\section{KN-9 Crystallographic studies on correlated electron systems under non-ambient conditions}

\author{
Karen Friese ${ }^{1}$ \\ 1. Jülich Centre for Neutron Science, Research Centre Jülich,
} Germany

email: k.friese@fz-juelich.de

Structural characteristics like symmetry, interatomic distances, or thermal movements of the atoms are closely related to the properties of a material. Correlated electron systems exhibiting novel magnetic properties, a superior optical and electric performance or superconductivity have excellent prospects as advanced materials. However, the establishment of reliable structure/property relationships is often difficult due to complications in the crystal structure determination process. This talk will concentrate on two classes of materials which are characterized by a strong coupling of charge, spin and/or lattice degrees of freedom. The first part will be centered on the behaviour of the crystal and magnetic structures of magnetocaloric compounds. In these materials, which can be used for advanced refrigeration technologies, the application of a magnetic fiels leads to changes in the magnetic entropy and adiabatic temperature. The magnetocaloric transition is usually accompanied by a structural transition and the response of the lattice to the onset of magnetic ordering is of key importance for the understanding of the underlying mechanism of the magnetocaloric effect. The second example will concentrate on mixed-valence compounds. Here, charge ordering can lead to significant changes of the coordination spheres of the aliovalent ions and lead to drastic changes in the underlying crystal structures often reflected in first-order phase transitions. Multiparametric studies on both systems performed at neutron and synchrotron source will be presented. Apart from the variation of temperature and chemical composition, hydrostatic pressure plays a key role in these studies as it permits to drastically influence the interatomic distances in the materials and can thus help to better understand the underlying mechanisms of the observed phenomena.

Keywords: Correlated electron systems, phase transitions, hydrostatic pressure, magnetic interactions, charge order

\section{KN-10 Advanced electron crystallography through imaging \\ Sandra Van Aert $^{1}$}

1. Electron Microscopy for Materials Science (EMAT), Universiteit Antwerpen, Belgium

email: sandra.vanaert@uantwerpen.be

New developments in the field of nanoscience and nanotechnology drive the need for advanced quantitative materials characterisation techniques that can be applied to complex nanostructures. The physical properties of these nanostructures are controlled by composition and chemical bonding, but also by the positions of the atoms. Indeed, changing the interatomic distances by picometers can turn an insulator into a conductor. Because of the presence of defects, interfaces and surfaces, the locations of atoms deviate from their equilibrium bulk positions giving rise to strain. In order to study nanostructures, transmission electron microscopy (TEM) is an excellent technique because of the strong interaction of electrons with small volumes of matter providing local information on the material under study. Over the past few years, remarkable high-technology developments in the lens design greatly improved the image resolution. Nowadays, a resolution of the order of $50 \mathrm{pm}$ can be achieved. For most atom types, this exceeds the point where the electrostatic potential of the atoms is the limiting factor. Furthermore, new data collection geometries are emerging that allow one to optimise the experimental settings. In addition, detectors behave more and more as ideal quantum detectors. In this manner, the microscope itself becomes less restricting and the quality of the experimental images is mainly set by the unavoidable presence of electron counting noise and environmental disturbances. In order to measure the atom positions and atom types as accurately and precisely as possible from atomic resolution TEM image, quantitative methods are required. To reach this goal, the use of statistical parameter estimation theory is of great help. This methodology allows one to measure 2D atomic column positions with subpicometer precision, to measure compositional changes at interfaces, to count atoms with single atom sensitivity, and to reconstruct $3 \mathrm{D}$ atomic structures. Using current state-of-the-art experimental examples, it will be shown how statistical parameter estimation techniques can be used to overcome the traditional limits set by modern TEM. The precision that can be achieved in this quantitative manner far exceeds the resolution performance of the instrument. This opens up a whole new range of possibilities to understand and characterise nanostructures at the atomic level and to help developing innovative materials with revolutionary interesting properties.

Keywords: electron microscopy, quantitative imaging 